\title{
Severe Neurotoxicity Following Ingestion of Tetraethyl Lead
}

\author{
Brandon K. Wills • Jason Christensen • \\ Joe Mazzoncini • Michael Miller
}

Published online: 20 March 2010

(C) American College of Medical Toxicology 2010

\begin{abstract}
Organic lead compounds are potent neurotoxins which can result in death even from small exposures. Traditionally, these compounds are found in fuel stabilizers, anti-knock agents, and leaded gasoline. Cases of acute organic lead intoxication have not been reported for several decades. We report a case of a 13-year-old Iraqi male who unintentionally ingested a fuel stabilizer containing $80-90 \%$ tetraethyl lead, managed at our combat support hospital. The patient developed severe neurologic symptoms including agitation, hallucinations, weakness, and tremor. These symptoms were refractory to escalating doses of benzodiazepines and ultimately required endotracheal intubation and a propofol infusion. Adjunctive therapies included chelation, baclofen, and nutrition provided through a gastrostomy tube. The patient slowly recovered and was discharged in a wheelchair 20 days after ingestion, still requiring tube feeding. Follow-up at 62 days post-ingestion revealed near-resolution of symp-
\end{abstract}

Presented at the Acute and Intensive Care Symposium, North American Congress of Clinical Toxicology Annual Meeting, September 14, 2008, Toronto, Canada.

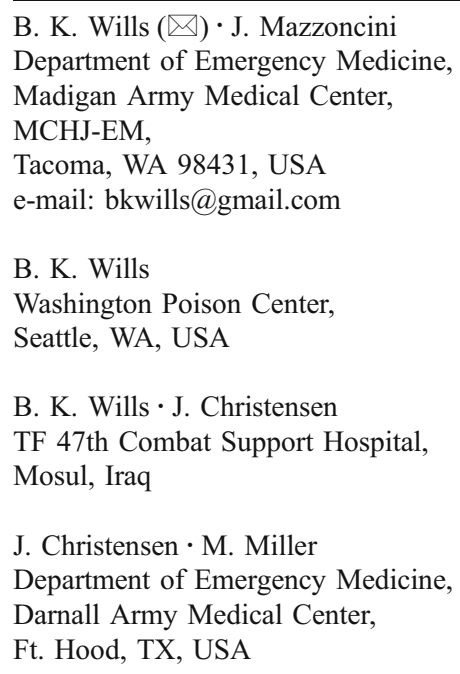

toms with residual slurred speech and slight limp. This case highlights the profound neurotoxic manifestations of acute organic lead compounds.

Keywords Organic lead · Tetraethyl lead · Poisoning · Chelation

\section{Introduction}

Organic lead compounds are primarily used in petroleum formulations as a stabilizer or "anti-knock" agent. Acute toxicity of organic lead compounds results in a syndrome distinct from the more commonly known inorganic lead toxicity. We had the opportunity to care for a 13-year-old Iraqi male who unintentionally ingested a mouthful of a fuel stabilizer resulting in severe neurologic sequelae. We describe the clinical course, treatment, and discussion of tetraethyl lead toxicity.

\section{Case Report}

A 13-year-old Iraqi male unintentionally ingested a mouthful of a gasoline additive containing $80-90 \%$ tetra ethyl lead, with dibromomethane and dichloromethane, stored in a juice container. Within minutes, he began vomiting and experiencing diffuse abdominal pain. He was taken to a local Iraqi hospital where he was evaluated and discharged home. Over the next $48 \mathrm{~h}$, he developed progressive tremors, hallucinations, and generalized weakness. He returned to the Iraqi hospital where he was admitted overnight and given intravenous (IV) $10 \%$ mannitol $300 \mathrm{~g}$, valium $5 \mathrm{mg}$ IV, furosemide $20 \mathrm{mg}$ IV, dexamethasone $16 \mathrm{mg}$ intramuscular, and propranolol $10 \mathrm{mg}$ orally prior to transfer to a United States Army Combat Support Hospital (CSH). 
The child was brought to the $\mathrm{CSH}$ on post-ingestion day 3. Initial examination revealed the following: temperature of $97.6^{\circ} \mathrm{F}$, heart rate of 70 beats per minute, blood pressure of 100/63 mmHg, respiratory rate of 20 breaths per minute, and oxygen saturation of $97 \%$ on room air. The patient was alert and oriented to self; he had slurred speech, episodic tremors, and intermittent visual hallucinations of being attacked by dogs and snakes. He was generally weak and unable to stand. Further assessment revealed normal cranial nerve function, global but non-focal muscular weakness, and symmetric $2 / 4$ brachioradialis, patellar, and Achilles deep tendon reflexes. The cardiopulmonary examination was normal: there were no external signs of trauma, and he had an otherwise unremarkable physical examination.

Initial laboratory values included: white blood cell count $11.1 \times 10^{9}$ cells $/ \mathrm{L}$; hemoglobin $124 \mathrm{~g} / \mathrm{L}$; platelet count $359 \times$ $10^{9} / \mathrm{L}$; sodium $135 \mathrm{mmol} / \mathrm{L}$; potassium $3.3 \mathrm{mmol} / \mathrm{L}$; chloride $95 \mathrm{mmol} / \mathrm{L}$; bicarbonate $27 \mathrm{mmol} / \mathrm{L}$; blood urea nitrogen $4.6 \mathrm{mmol} / \mathrm{L}$; creatinine $44.2 \mu \mathrm{mol} / \mathrm{L}$; glucose $7.9 \mathrm{mmol} / \mathrm{L}$; alkaline phosphatase $3.1 \mu \mathrm{kat} / \mathrm{L}$; alanine aminotransferase $0.89 \mu \mathrm{kat} / \mathrm{L}$; aspartate aminotransferase $1.12 \mu \mathrm{kat} / \mathrm{L}$; and serum iron $10.9 \mu \mathrm{mol} / \mathrm{L}$. Other liver function tests, coagulation studies, and urinalysis were obtained, and all results were within the normal reference ranges. The whole blood lead level on post-ingestion day 3 was $62 \mathrm{mcg} / \mathrm{dL}$. Additional whole blood and 24-h urine lead levels were sent during this hospitalization and on follow-up visits (Fig. 1). Non-contrast abdominal and head computed tomography (CT) on post-ingestion day 3 was unremarkable.

Although the exact nature of the substance was unknown at the time, fuel stabilizers are known to contain organic lead so chelation therapy was initiated. British anti-Lewisite (BAL) and succimer were unavailable; therefore, the patient was treated with calcium disodium ethylenediaminetetraacetic acid (EDTA), $1 \mathrm{~g}$ IV over $8 \mathrm{~h}$ daily for 5 days. Whole blood and 24-h urine levels were sent to the United States, but results were not rapidly available (Fig. 1). The patient had increasing agitation and confusion with worsening hallucinations, severe rhythmic myoclonus, and hyperreflexia. These symptoms were treated with periodic doses of lorazepam with minimal effect. A lumbar puncture was performed on postingestion day 5, and empiric ceftriaxone and acyclovir were added to cover for possible herpes encephalitis or meningitis. By post-ingestion day 6 , he continued to require escalating doses of benzodiazepines, ultimately necessitating endotracheal intubation and propofol infusion to control severe neuromuscular symptoms. Due to concerns about the patient being on a prolonged propofol infusion, benzodiazepines and baclofen were used concomitantly in order to wean the propofol. After 2 days of mechanical ventilation, the patients' symptoms began to improve and he was successfully extubated. After extubation, neuromuscular symptoms persisted, including severe hallucinations, weakness, tremor, and hyperreflexia. Dysphagia prevented adequate swallowing; therefore, a percutaneous gastrostomy tube was placed. Creatine phosphokinase peaked on day 8 at $10.6 \mu \mathrm{kat} / \mathrm{L}$. Repeat head CT on post-ingestion day 15 using imaging parameters identical to those used for the original scan revealed globally decreased attenuation of the white matter without mass effect. This appearance was felt to be compatible with an acute or subacute toxic demyelinating process (Figs. 2 and 3). He was discharged from the CSH on post-ingestion day 20 with persistent but improving hallucinations, generalized weakness, inability to ambulate, and urinary and fecal incontinence.

Analysis of the ingested substance was performed in the United States using Fourier transform infrared spectroscopy and gas chromatography/mass spectroscopy. Composition of the sample included: tetraethyl lead 80-90\%, dichloroethane $5-10 \%$, dibromoethane $5-10 \%$, gasoline $\sim 1 \%$, and minor components $<0.1 \%$ (tetramethyl lead, triethylmethyl lead, chloroethane, and bromoethane).

The patient returned to the $\mathrm{CSH}$ for follow-up on postingestion days $32,46,62$, and 91 . At the post-ingestion day 32 follow-up, his hallucinations were present only at night; his urinary incontinence was resolved, but his fecal

Fig. 1 Event timeline by number of days after ingestion of tetraethyl lead. Combat Support Hospital (CSH); PbB (whole blood lead level, micrograms per deciliter); $\mathrm{PbU}$ (24-h urine lead level, micrograms per liter); ethylenediaminetetraacetic acid (EDTA); computed tomography (CT); lumbar puncture (LP); ceftriaxone (CTX)

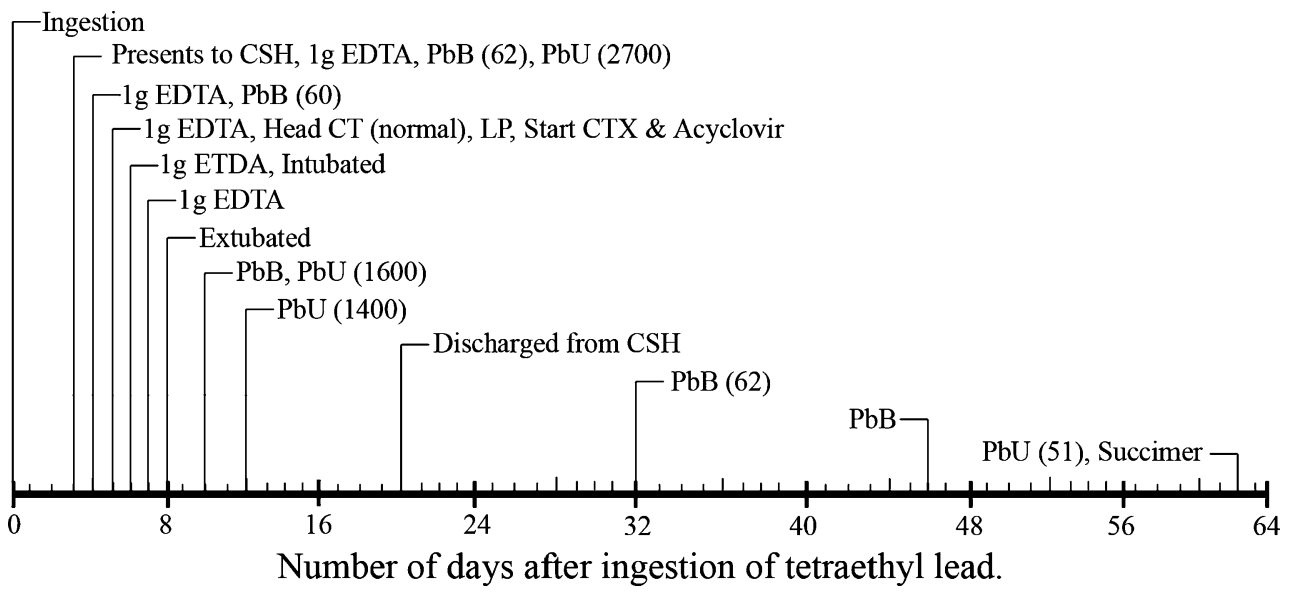




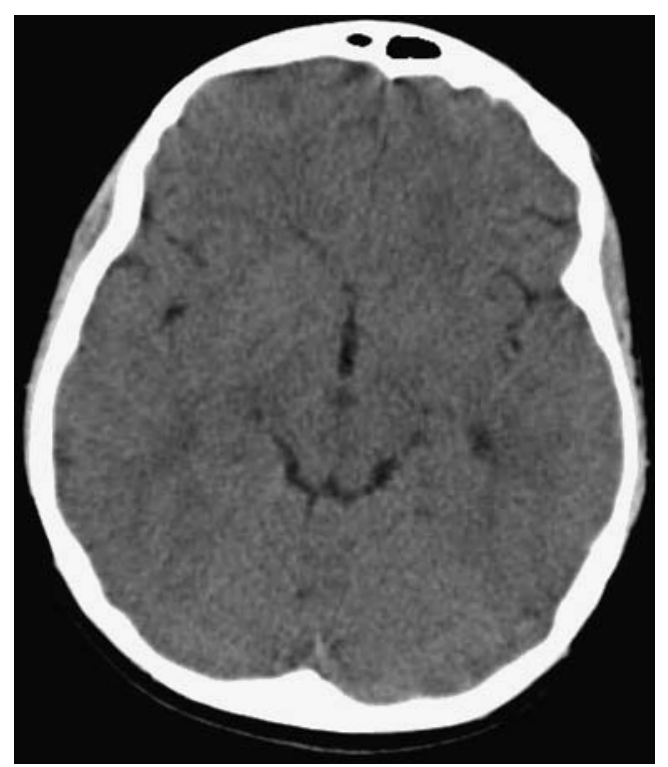

Fig. 2 Normal non-contrast computed tomography of the head on post-ingestion day 5

incontinence persisted. The patient continued to require tube feeding. His neurologic examination revealed improved alertness, ability to verbalize with his family, intermittent oral muscle twitching and smacking, 3/4 brachioradialis, patellar, and Achilles deep tendon reflexes, improved strength and finger-to-nose coordination, and ability to stand but not ambulate. At the post-ingestion day 62 follow-up visit, the patient was fully continent and able to verbalize, tolerate oral nutrition, ambulate, and perform his activities of daily living without assistance. Repeat neurologic examination at that time revealed further serial improvement, with only some residual deficits including a slight limp and mild slurring of his speech.

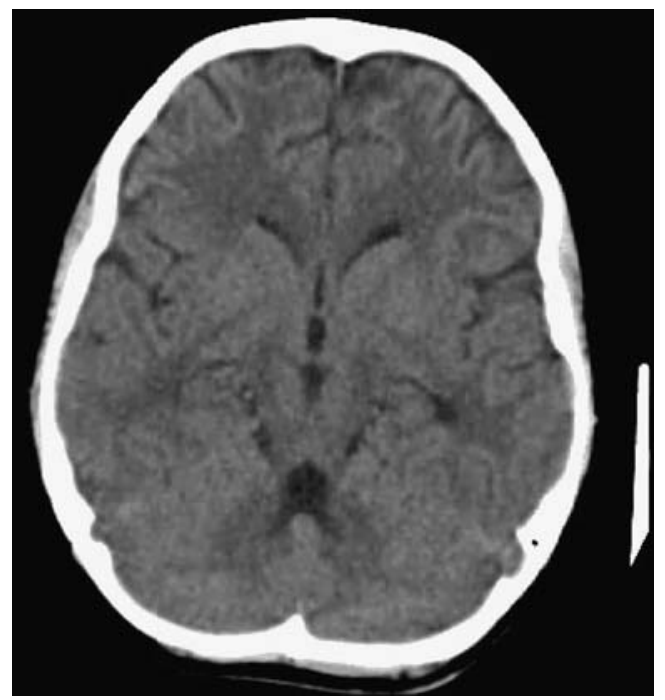

Fig. 3 Non-contrast computed tomography of the head on postingestion day 15 , revealing diffuse demyelinization
By this visit, we had obtained succimer from the United States and elected to initiate therapy. Succimer was administered $10 \mathrm{mg} / \mathrm{kg}$ PO TID for 5 days, then $10 \mathrm{mg} / \mathrm{kg}$ PO BID for 14 days. Another whole blood lead was sent at this visit and was $51 \mathrm{mcg} / \mathrm{dl}$ (Fig. 1). His final visit occurred on post-ingestion day 91 . He continued to do well. His speech had normalized. His father stated that the patient had some difficulties focusing at times. His gait abnormality had subsided, and he had resumed riding his bike.

\section{Discussion}

Organic lead compounds including tetraethyl lead have been traditionally used as octane boosting and anti-knock additives. Since the Clean Air Act of 1990 [1], tetraethyl lead is no longer routinely available in the United States. The epidemiology of acute tetraethyl lead intoxication is not wellstudied since these exposures are not tracked by the American Association of Poison Control Centers' National Poison Data Base (NPDB) [2]; however, cases have rarely been reported in the medical literature since the 1970s [3].

Organic lead compounds are potent neurotoxins. In rats, the $\mathrm{LD}_{50}$ 's are approximately 11.2 and $15.4 \mathrm{mg} / \mathrm{kg}$ for triethyl and tetraethyl lead, respectively [4]. Unlike inorganic lead, organolead compounds have a distinct toxic syndrome which reflects their unique neurotoxic pathophysiology. Signs of acute intoxication include: lethargy, tremor, increased muscle tone, exaggerated tendon reflexes, psychosis, and encephalopathy which can progress to convulsions, paralysis, and death [3, 5-7]. Chronic organolead exposure from huffing leaded gasoline can result in persistent neuropsychiatric symptoms including: encephalopathy, dementia, cerebellar signs, and organic psychosis $[7,8]$.

Tetraethyl lead is dealkylated in liver microsomes to triethyl lead [9] which is considered the primary neurotoxic metabolite. There is some evidence to suggest that organolead compounds are further dealkylated in vivo to inorganic lead [10]. In a rat model, subcutaneous administration of triethyl lead results in a 100-fold increase in blood lead compared with a 20 -fold increase in the brain [11]. The elimination half-lives in blood and brain in rats are approximately 5 and 8 days, respectively [11] but may be much longer in humans [7].

Histologic changes of the brain occur primarily in the limbic system and frontal cortex and include: loss of neurons, spongiose transformation of white matter, and myelinolysis [3, 5]. Neuronal necrosis has also been documented in the brainstem, midbrain, pontine, basal, and anterior cervical spinal cord nuclei $[6,12]$.

Triethyl lead exposure results in mitochondrial damage, impaired brain oxygen consumption, and accumulation of lactic acid $[6,9,12,13]$. Organolead exposure also inhibits 
dopamine, GABA, and glutamate uptake [5] and results in exaggerated motor responses to dopaminergic agents which may explain the neuromuscular effects seen with toxicity [11]. The successful attenuation of hallucinations induced by huffing leaded gasoline through treatment with haloperidol also supports the theory that organolead's hallucinogenic effects are mediated by dopaminergic pathways $[8,11]$.

The therapy for acute organolead intoxication consists primarily of supportive measures because chelation is unlikely to influence outcome. Patients may need considerable sedation to prevent complications associated with a hypermetabolic state. Patients presenting to a healthcare facility early $(<1 \mathrm{~h})$ after ingestion may be considered for nasogastric aspiration. Activated charcoal is unstudied but unlikely to be effective, increases the risk of pulmonary aspiration, and is not recommended for most hydrocarbon ingestions. There are no readily available assays for organolead compounds. Whole blood lead levels will be elevated and may confirm exposure to an organolead compound; however, these levels do not distinguish between organic and inorganic forms of lead.

Chelation therapy is of unknown benefit but is unlikely to be useful. Tetraethyl lead has all valencies satisfied and forms lead salts following dealkylation. None of the alkyl lead forms appear to interact with traditional chelators [7, 9]. Two fatal tetraethyl lead ingestions treated with EDTA had no apparent clinical effect [3]. In animal models, EDTA and BAL were ineffective at chelating triethyl lead $[9,13]$. Chronic tetraethyl lead intoxication from huffing leaded gasoline has also been treated with BAL and EDTA resulting in blood lead level reductions similar to endogenous clearance alone $[8,14]$. In one series of patients with neurotoxicity from huffing gasoline, treatment with BAL and EDTA and was reported to decrease whole blood lead concentrations during therapy [15]. The theoretic utility of chelation therapy would be to bind a small percentage of biotransformed inorganic lead; however, this therapy is of unproven clinical efficacy and is generally not recommended. Given the devastating nature of acute tetraethyl lead toxicity; however, chelation therapy may be considered.

In this case report, the clinical and temporal presentation is typical for acute organic lead toxicity [3]. Most organic lead exposures have traditionally been from leaded gasoline huffing. The pathophysiology in these cases may be different due to chronic, repeated exposures as well as the presence of other hydrocarbons which could contribute to encephalopathy. It is also common in patients who huff leaded gasoline to develop chronic neuropsychiatric symptoms [7, 8, 14, 15]. The only series of acute oral ingestion of tetraethyl lead described two fatalities from small volume intentional exposures [3]. To our knowledge, recovery from acute oral exposure to organic lead has not been reported.

\section{Conclusion}

A case of acute organic lead ingestion is reported resulting in severe neurologic impairment as manifested by mental status depression, hallucinations, hyperexcitability, and rhythmic myoclonus. Its treatment focused on aggressive supportive measures. Acute organic lead toxicity is rare in the United States but may be seen by clinicians practicing internationally. Despite severe neurotoxicity, near-complete recovery occurred in this case.

Acknowledgement The opinion and assertions contained herein are the views of the author and are not to be construed as official or as reflecting the views of the United States Department of Defense.

The authors would like to express our gratitude to Bijan Saeedi at S.E.T Environmental Inc. for graciously analyzing the fuel stabilizer.

\section{References}

1. Environmental Protection Agency. www.epa.gov. Accessed 12/ 2006

2. American Association of Poison Control Centers. National Poison Data Base. www.aapcc.org. Accessed 12/2006

3. Forycki Z, Zegarski W, Bardzik J (1977) Two cases of suicidal tetraethyl lead poisoning. Bull Inst Marit Trop Med Gdynia 28(3-4):179-185

4. Cremer JE (1961) The toxicity of tetraethyl lead and related alkyl metallic compounds. Ann Occup Hyg 3:226-230

5. Verity MA (1990) Comparative observations on inorganic and organic lead neurotoxicity. Environ Health Perspect 89:43-48

6. Chang LW (1990) The neurotoxicology and pathology of organomercury, organolead, and organotin. J Toxicol Sci 15(Suppl 4):125151

7. Tenenbein M (1997) Leaded gasoline abuse: the role of tetraethyl lead. Hum Exp Toxicol 16(4):217-222

8. Goldings AS, Stewart RM (1982) Organic lead encephalopathy: behavioral change and movement disorder following gasoline inhalation. J Clin Psychiatry 43(2):70-72

9. Cremer JE (1959) Biochemical studies on the toxicity of tetraethyl lead and other organo-lead compounds. Br J Ind Med 16:191-199

10. Arai F, Yamamura Y, Yoshida M (1981) Excretion of triethyl lead, diethyl lead and inorganic lead after injection of tetraethyl lead in rabbits (author's transl). Sangyo Igaku 23(5):496-504

11. Walsh TJ, Schulz DW, Tilson HA, Dehaven DL (1986) Acute exposure to triethyl lead enhances the behavioral effects of dopaminergic agonists: involvement of brain dopamine in organolead neurotoxicity. Brain Res 363(2):222-229

12. Walsh TJ, McLamb RL, Bondy SC, Tilson HA, Chang LW (1986) Triethyl and trimethyl lead: effects on behavior, CNS morphology and concentrations of lead in blood and brain of rat. Neurotoxicology 7(3):21-33

13. Cremer JE, Vardanis A, Quastel JH (1962) Tetraethyl lead toxicity in rats. Nature 195:607-608

14. Robinson RO (1978) Tetraethyl lead poisoning from gasoline sniffing. JAMA 240(13):1373-1374

15. Burns CB, Currie B (1995) The efficacy of chelation therapy and factors influencing mortality in lead intoxicated petrol sniffers. Aust N Z J Med 25(3):197-203 